\title{
Time Delay Analysis of Turbofan Engine Direct and Indirect Combustion Noise Sources
}

\author{
(AIAA-2008-50 Spectral Separation of the \\ Turbofan Engine Coherent Combustion Noise Component)
}

AARC Turbine Noise Workshop. Vancouver, BC, May 8-9,2008

JEFFREY HILTON MILES*

NASA John H. Glenn Research Center at Lewis Field, Cleveland, OH 44135

The core noise components of a dual spool turbofan engine were separated by the use of a coherence function. A source location technique based on adjusting the time delay between the combustor pressure sensor signal and the far-field microphone signal to maximize the coherence and remove as much variation of the phase angle with frequency as possible was used. The discovery was made that for the $130^{\circ}$ microphone a $90.027 \mathrm{~ms}$ time shift worked best for the frequency band from 0 to $200 \mathrm{~Hz}$ while a $86.975 \mathrm{~ms}$ time shift worked best for the frequency band from 200 to $400 \mathrm{~Hz}$. Hence, the 0 to $200 \mathrm{~Hz}$ band signal took more time than the 200 to $400 \mathrm{~Hz}$ band signal to travel the same distance. This suggests the 0 to $200 \mathrm{~Hz}$ coherent cross spectral density band is partly due to indirect combustion noise attributed to entropy fluctuations, which travel at the flow velocity, interacting with the turbine. The signal in the 200 to $400 \mathrm{~Hz}$ frequency band is attributed mostly to direct combustion noise. Results are presented herein for engine power settings of 48,54, and 60 percent of the maximum power setting.

*Aerospace Engineer, Acoustics Branch, 21000 Brookpark Road, Cleveland, Ohio, 44135, 


\title{
Time Delay Analysis of Turbofan Engine Direct and Indirect Combustion Noise Sources
}

(AIAA-2008-50 Spectral Separation of the Turbofan

\author{
Engine Coherent Combustion Noise Component)
}

\author{
Jeffrey Hilton Miles
}

National Aeronautics and Space Administration

Glenn Research Center at Lewis Field, Cleveland, Ohio 44135 


\section{INTRODUCTION}

- Engine Combustion-Noise Sources

$\diamond$ direct: pressure fluctuations directly produced by unsteady combustion

$\diamond$ indirect: entropy fluctuations converted to noise by turbine stage

- Diagnostic Technique

$\diamond$ coherence analysis with adjustable time delay

$\diamond$ maximize coherence-minimize phase angle variation

- NASA/Honeywell EVNERT Data

$\diamond$ Honeywell Aerospace's San Tan outdoor engine test facility, 2005-2007

$\diamond$ Honeywell TECH977 research engine typical of $6,000-8,000$ lbs thrust class

$\diamond$ far-field microphones \& internal sensors

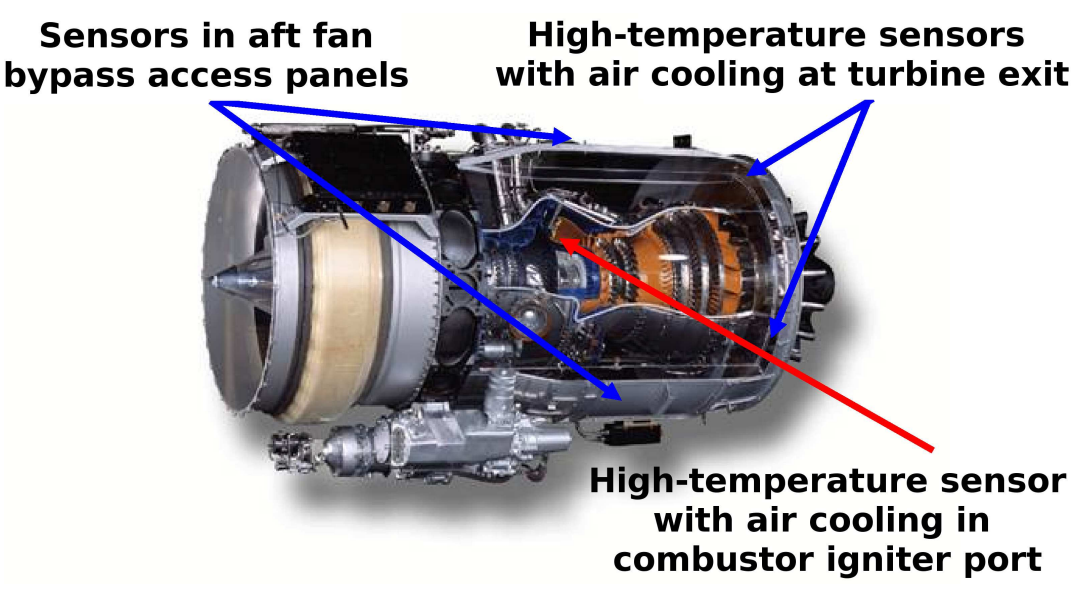




\section{COHERENCE FUNCTION}

- Coherence Function, $\gamma(f)$ :

$$
\begin{gathered}
\gamma_{x y}^{2}(f)=\frac{\left|G_{x y}(f)\right|^{2}}{G_{x x}(f) G_{y y}(f)} \quad 0 \leq \gamma_{x y}^{2} \leq 1 \\
G_{x y}(f)=\text { cross power spectral density }
\end{gathered}
$$

- Finite Data Sequences $\Rightarrow$ Noise-Floor Concept

$$
\begin{gathered}
\gamma_{n n}^{2}<\gamma_{x y}^{2} \leq 1 \quad \gamma_{n n}^{2}=1-(1-P)^{1 /(M-1)} \\
P=0.95 \& M=1120 \Rightarrow \gamma_{n n}^{2}=0.00267
\end{gathered}
$$

$P=$ P-percent confidence interval $\quad M=$ number of independent data segments

$\diamond$ traditionally: $\gamma_{n n}^{2}=0.1$

- Time-Shift $(D)$ Far-Field Signal To Account For Propagation Delay

$\diamond$ unaligned $(D=0)$ : only tones above noise floor

$\diamond$ aligned $(D>0)$ : coherent broadband also above noise floor select $D$ to minimize variation in $\arg \left[G_{x y}(f ; D)\right]$ 


\section{4 \% POWER, 130 DEG. DIRECTION}

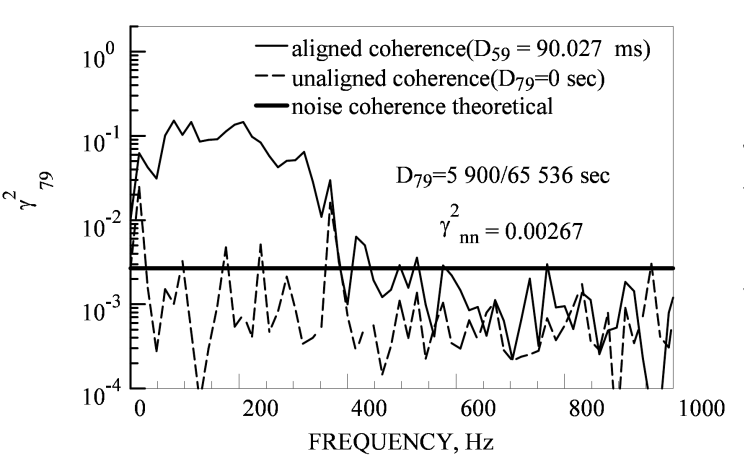

(a) Coherence between microphone at $130^{\circ}$ and $\mathrm{C} 1 \mathrm{P} 1$ combustor pressure sensor.

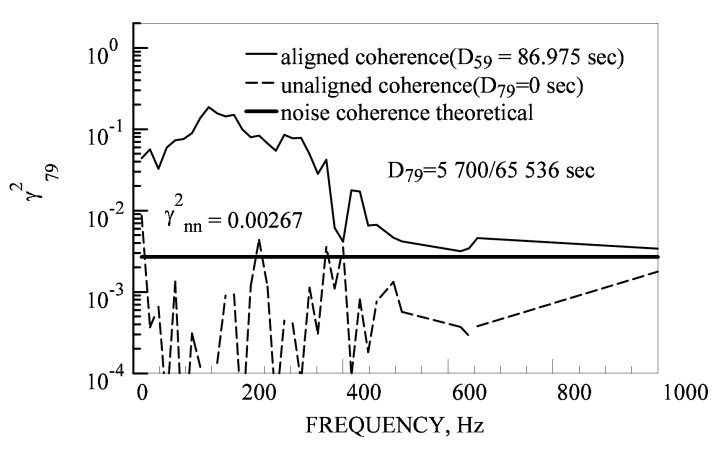

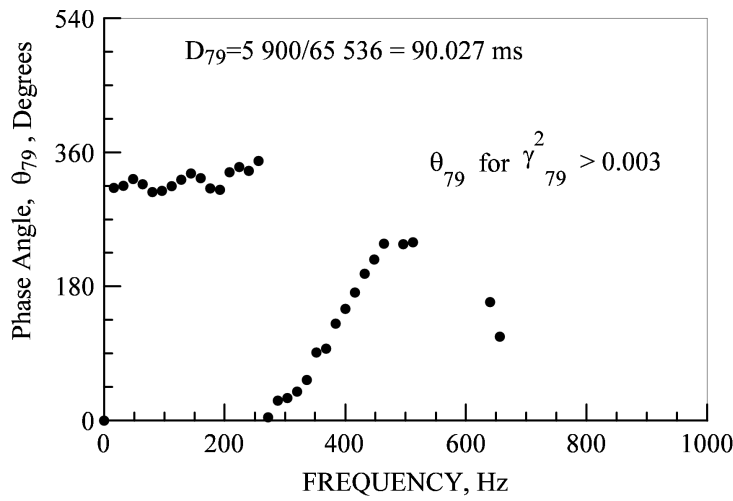

(b) Aligned cross-spectrum phase angle between microphone at $130^{\circ}$ and $\mathrm{C} 1 \mathrm{P} 1$ combustor pressure sensor.

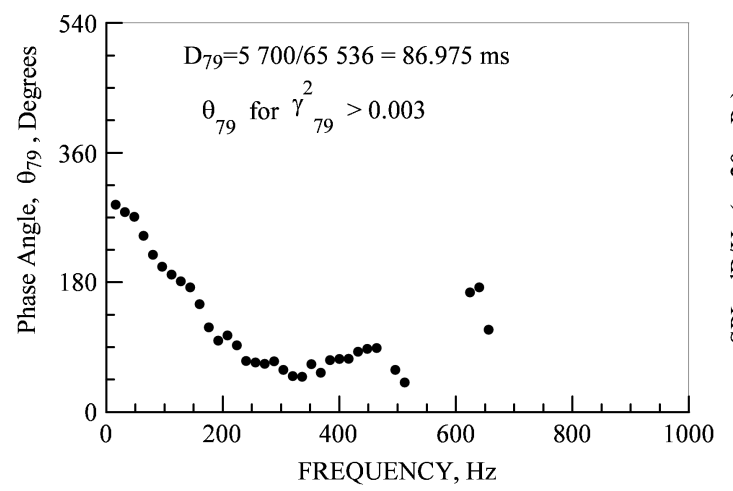

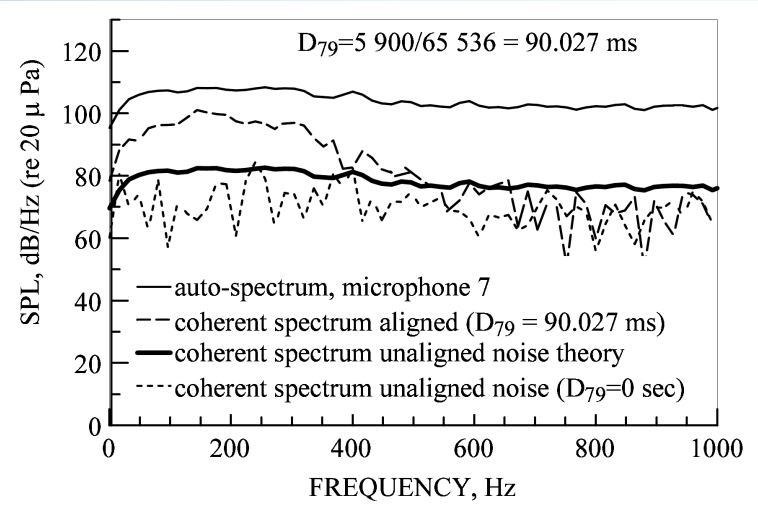

(c) Coherent output power at microphone at $130^{\circ}$ using C1P1 combustor pressure sensor.

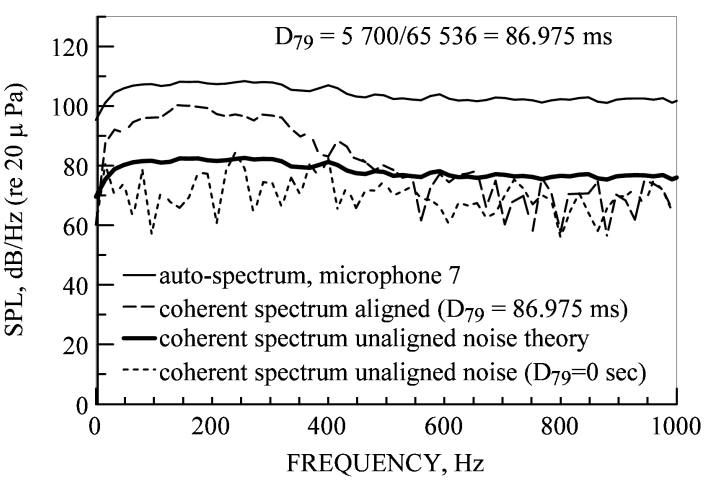

- Coherent Signal In 0-200 Hz Band Took 3.05 ms Longer Time Than 200-400 Hz Band

$\diamond$ Same $\Delta t$ for all power levels $(48,54,60 \%)$ and direction $(110,130,160$ deg.) 


\section{SUMMARY}

- Variable Time-Delay Coherence Analysis of Honeywell/NASA

EVNERT Static Engine-Test Data

$\diamond$ internal pressure sensor \& aft-quadrant far-field microphones

$\diamond$ technique maximizes coherence and minimizes phase-angle variation

$\diamond$ coherence noise floor is identified

- Aligned And Unaligned Coherence Techniques Used

$\diamond$ method was successful

$\diamond$ low levels of coherence validated as due to combustion noise

- Strong Indication of Indirect Combustion Noise

$\diamond$ coherent noise in the $0-200 \mathrm{~Hz}$ frequency band had longer propagation time than the $200-400 \mathrm{~Hz}$ band

$\diamond$ direct combustion noise propagates at the local speed of sound everywhere

$\diamond$ indirect combustion noise propagates as entropy fluctuations with the local mean flow velocity until converted to acoustic waves in the turbine

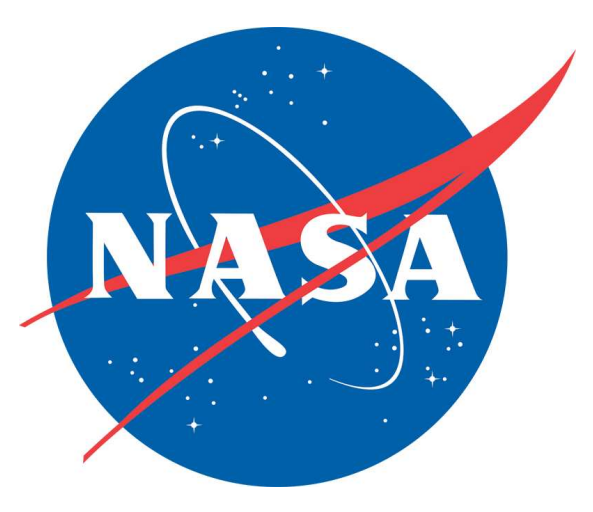
$\Rightarrow$ should take longer time to reach far-field microphone. 